\title{
Front Matter: Volume 7832
}

, "Front Matter: Volume 7832," Proc. SPIE 7832, Lidar Technologies, Techniques, and Measurements for Atmospheric Remote Sensing VI, 783201 (30 November 2010); doi: 10.1117/12.882322

SPIE. Event: SPIE Remote Sensing, 2010, Toulouse, France 


\section{PROCEEDINGS OF SPIE}

\section{Lidar Technologies, Techniques, and Measurements for Atmospheric Remote Sensing VI}

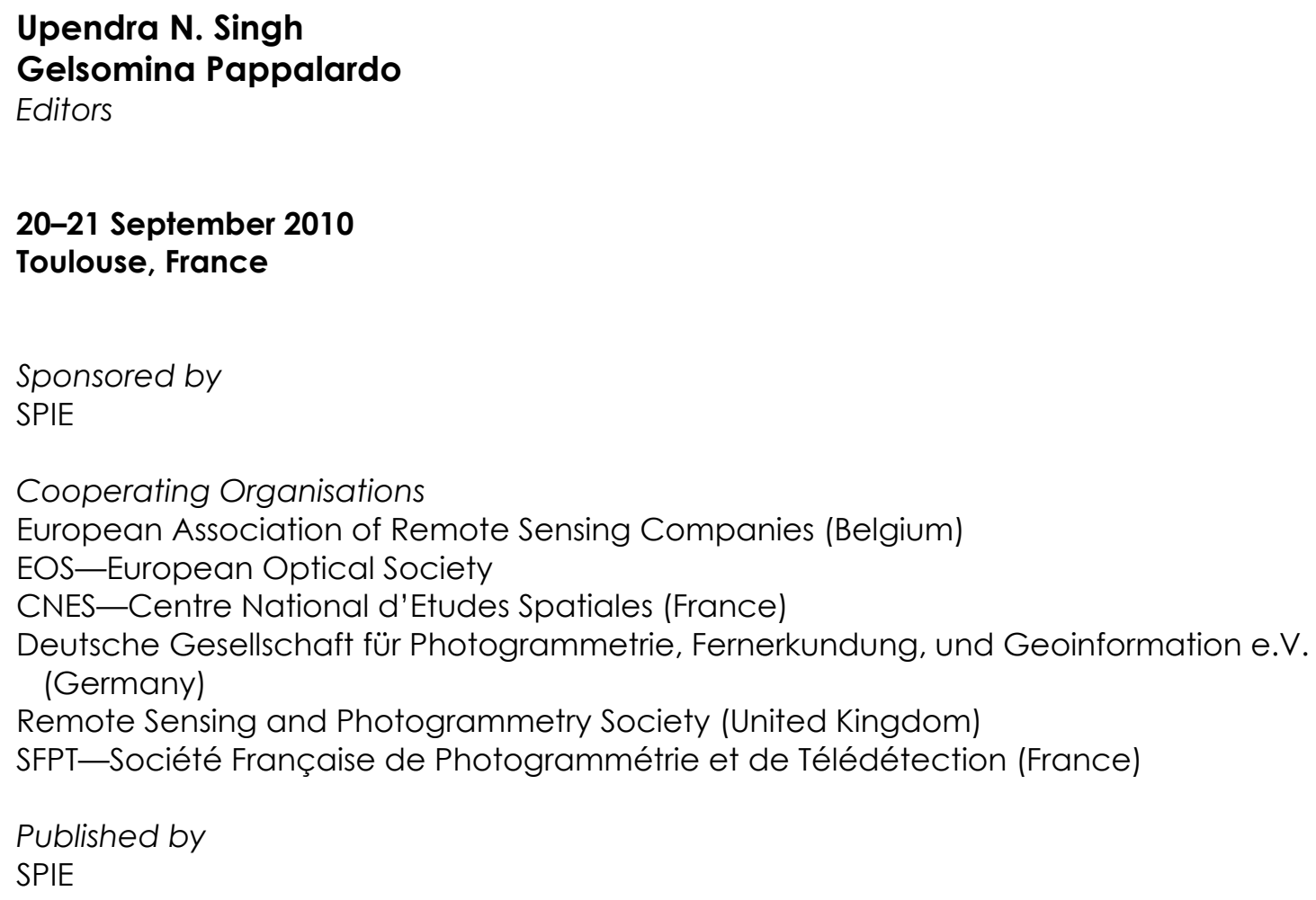

Volume 7832 
The papers included in this volume were part of the technical conference cited on the cover and title page. Papers were selected and subject to review by the editors and conference program committee. Some conference presentations may not be available for publication. The papers published in these proceedings reflect the work and thoughts of the authors and are published herein as submitted. The publisher is not responsible for the validity of the information or for any outcomes resulting from reliance thereon.

Please use the following format to cite material from this book:

Author(s), "Title of Paper," in Lidar Technologies, Techniques, and Measurements for Atmospheric Remote Sensing VI, edited by Upendra N. Singh, Gelsomina Pappalardo, Proceedings of SPIE Vol. 7832 (SPIE, Bellingham, WA, 2010) Article CID Number.

ISSN 0277-786X

ISBN 9780819483492

Published by

SPIE

P.O. Box 10, Bellingham, Washington 98227-0010 USA

Telephone +1 3606763290 (Pacific Time) · Fax +1 3606471445

SPIE.org

Copyright (C) 2010, Society of Photo-Optical Instrumentation Engineers

Copying of material in this book for internal or personal use, or for the internal or personal use of specific clients, beyond the fair use provisions granted by the U.S. Copyright Law is authorized by SPIE subject to payment of copying fees. The Transactional Reporting Service base fee for this volume is $\$ 18.00$ per article (or portion thereof), which should be paid directly to the Copyright Clearance Center (CCC), 222 Rosewood Drive, Danvers, MA 01923. Payment may also be made electronically through CCC Online at copyright.com. Other copying for republication, resale, advertising or promotion, or any form of systematic or multiple reproduction of any material in this book is prohibited except with permission in writing from the publisher. The CCC fee code is 0277-786X/10/\$18.00.

Printed in the United States of America.

Publication of record for individual papers is online in the SPIE Digital Library.

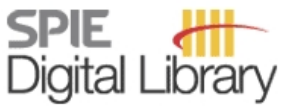

SPIEDigitalLibrary.org

Paper Numbering: Proceedings of SPIE follow an e-First publication model, with papers published first online and then in print and on CD-ROM. Papers are published as they are submitted and meet publication criteria. A unique, consistent, permanent citation identifier (CID) number is assigned to each article at the time of the first publication. Utilization of CIDs allows articles to be fully citable as soon they are published online, and connects the same identifier to all online, print, and electronic versions of the publication. SPIE uses a six-digit CID article numbering system in which:

- The first four digits correspond to the SPIE volume number.

- The last two digits indicate publication order within the volume using a Base 36 numbering system employing both numerals and letters. These two-number sets start with 00, 01, 02, 03, 04, $05,06,07,08,09,0 A, 0 B \ldots 0 Z$, followed by 10-1Z, 20-2Z, etc.

The CID number appears on each page of the manuscript. The complete citation is used on the first page, and an abbreviated version on subsequent pages. Numbers in the index correspond to the last two digits of the six-digit CID number. 


\section{Contents}

vii Conference Committee

\section{SESSION 1 LIDAR TECHNIQUES}

783202 Advances in high-energy solid-state 2-micron laser transmitter development for ground and airborne wind and $\mathrm{CO}_{2}$ measurements (Invited Paper) [7832-01]

U. N. Singh, J. Yu, NASA Langley Research Ctr. (United States); M. Petros, Science and Technology Corp. (United States); S. Chen, M. J. Kavaya, B. Trieu, NASA Langley Research Ctr. (United States); Y. Bai, Science Systems and Applications, Inc. (United States); P. Petzar, National Institute of Aerospace (United States); E. A. Modlin, G. Koch, J. Beyon, NASA Langley Research Ctr. (United States)

783203 An automatic planetary boundary layer height detection with a compact aerosol UV lidar [7832-02]

L. Sauvage, S. Loaëc, S. Lolli, M. Boquet, A. El Filali, Leosphere France (France)

783205 Simulation of Doppler lidar measurements using the WRF and Yamada-Mellor models [7832-04]

E. A. Shelekhova, A. P. Shelekhov, V.E. Zuev Institute of Atmospheric Optics (Russian Federation); A. V. Starchenko, A. A. Barth, D. A. Belikov, Tomsk State Univ. (Russian

Federation)

783206 Development of a fiber-based eye safe coherent wind lidar system for urban wind field measurements [7832-05]

M. F. Arend, S. Abdelazim, D. Santoro, F. Moshary, B. Gross, S. Ahmed, The City College of New York (United States)

783207 ValidWind applications: wind power prospecting, aerosol transport [7832-06]

T. Wilkerson, A. Marchant, T. Apedaile, D. Scholes, J. Simmons, B. Bradford, Energy Dynamics Lab. (United States)

\section{SESSION 2 SPACE-BASED LIDAR}

7832 OB The CALIPSO Mission: results and progress (Invited Paper) [7832-09]

D. Winker, Y. Hu, M. Pitts, M. Avery, NASA Langley Research Ctr. (United States); B.

Getzewich, J. Tackett, C. Kittaka, Science Systems and Applications, Inc. (United States); Z. Liu, National Institute of Aerospace (United States); M. Vaughan, NASA Langley Research Ctr. (United States)

7832 OC PBL-height derivation from the CALIOP/CALIPSO and comparing with the radiosonde and ground-based lidar measurements [7832-10]

Y. Wu, C.-M. Gan, L. Cordero, B. Gross, F. Moshary, S. Ahmed, The City College of New York (United States) 
$7832 \mathrm{OD}$ A lidar approach to measure $\mathrm{CO}_{2}$ concentrations from space for the ASCENDS Mission [7832-11]

J. B. Abshire, H. Riris, NASA Goddard Space Flight Ctr. (United States); G. R. Allan, Sigma Space Inc. (United States); C. J. Weaver, J. Mao, Goddard Earth Sciences and Technology Ctr., UMBC (United States); X. Sun, NASA Goddard Space Flight Ctr. (United States);

W. E. Hasselbrack, Sigma Space Inc. (United States); A. YU, Goddard Earth Sciences and Technology Ctr., UMBC (United States); A. Amediek, Deutsches Zentrum für Luft- und Raumfahrt e.V. (Germany); Y. Choi, E. V. Browell, NASA Langley Research Ctr. (United States)

\section{SESSION 3 WATER VAPOR, AEROSOL, AND CLOUDS}

7832 OE Water vapour profiling in cloudy conditions integrating Raman lidar and passive microwave observations [7832-12]

F. Madonna, A. Boselli, A. Amodeo, C. Cornacchia, G. D'Amico, A. Giunta, L. Mona, G. Pappalardo, Istituto di Metodologie per l'Analisi Ambientale, CNR (Italy)

7832 OF Indirect aerosol hygroscopic growth observations with a backscatter lidar [7832-13] P. F. Rodrigues, F. S. Lopes, R. F. da Costa, W. M. Nakaema, E. Landulfo, Instituto de Pesquisas Energéticas e Nucleares (Brazil)

7832 OG Potential investigation of cloud-aerosol interaction with a multiple-wavelength Ramanelastic lidar [7832-14]

Y. Wu, L. Cordero, C.-M. Gan, B. Gross, F. Moshary, S. Ahmed, The City College of New York (United States)

$7832 \mathrm{OH}$ Integration of remote lidar and in-situ measured data to estimate particulate flux and emission from tillage operations [7832-15]

V. V. Zavyalov, G. E. Bingham, M. Wojcik, Space Dynamics Lab. (United States); J. L. Hatfield, Agricultural Research Service (United States); T. D. Wilkerson, Space Dynamics Lab. (United States); R. S. Martin, Utah State Univ. (United States); C. Marchant, K. Moore, B. Bradford, Space Dynamics Lab. (United States)

7832 ol Lidar observation campaign of sugar cane fires and industrial emissions in the state of São Paulo, Brazil [7832-16]

E. Landulfo, Instituto de Pesquisas Energéticas e Nucleares (Brazil); M. P. M. P. Jorge, Ctr. de Previsão de Tempo e Estudos Climáticos (Brazil); G. Held, Univ. Estadual Paulista Júlio de Mesquita Filho (Brazil); R. Guardani, J. Steffens, Escola Politécnica da Univ. de São Paulo (Brazil); S. dos Anjos F. Pinto, I. R. Andre, G. Garcia, Univ. Estadual Paulista - Rio Claro (Brazil); F. J. S. Lopes, Instituto de Pesquisas Energéticas e Nucleares (Brazil); G. L. Mariano, Ctr. de Previsão de Tempo e Estudos Climáticos (Brazil); R. F. da Costa, P. F. Rodrigues, Instituto de Pesquisas Energéticas e Nucleares (Brazil)

JOINT SESSION WITH CONFERENCE 7827: LIDAR MEASUREMENTS DURING RECENT 2010 EYJAFJALLAJÖKULL VOLCANIC ERUPTION

7832 0J EARLINET observations of the Eyjafjallajökull ash plume over Europe (Invited Paper) [7832-17] G. Pappalardo, A. Amodeo, Istituto di Metodologie per I'Analisi Ambientale, CNR (Italy); A. Ansmann, Leibniz Institut für Troposphärenforschung (Germany); A. Apituley, Rijksinstituut voor Volksgezondheid en Milieu (Netherlands); L. Alados Arboledas, Univ. de Granada (Spain); D. Balis, Aristoteleio Panepistimio Thessaloniki (Greece); C. Böckmann, Univ. 
Potsdam (Germany); A. Chaikovsky, Institute of Physics (Belarus); A. Comeron, Univ. Politècnica de Catalunya (Spain); G. D'Amico, Istituto di Metodologie per l'Analisi Ambientale, CNR (Italy); F. De Tomasi, Univ. del Salento (Italy); V. Freudenthaler, LudwigMaximilians-Univ. München (Germany); E. Giannakaki, Aristoteleio Panepistimio Thessaloniki (Greece); A. Giunta, Istituto di Metodologie per I'Analisi Ambientale, CNR (Italy); I. Grigorov, Institute of Electronics (Bulgaria); O. Gustafsson, Swedish Defence Research Agency (Sweden); S. Gross, Ludwig-Maximilians-Univ. München (Germany); M. Haeffelin, Univ. Pierre et Marie Curie (France); M. Iarlori, Univ. degli Studi dell'Aquila (Italy); S. Kinne, H. Linné, Max-Planck-Institut für Meteorologie (Germany); F. Madonna, Istituto di Metodologie per I'Analisi Ambientale, CNR (Italy); R. Mamouri, National Technical Univ. of Athens (Greece); I. Mattis, Leibniz Institut für Troposphärenforschung (Germany); M. McAuliffe, Univ. College Cork (Ireland); F. Molero, Ctr. de Investigaciones Energéticas, Medioambientales y Tecnológicas (Spain); L. Mona, Istituto di Metodologie per l'Analisi Ambientale, CNR (Italy); D. Müller, Leibniz Institut für Troposphärenforschung (Germany); V. Mitev, Ctr. Suisse d'Electronique et de Microtechnique SA (Switzerland); D. Nicolae, National Institute of Research and Development for Optoelectronics (Romania); A. Papayannis, National Technical Univ. of Athens (Greece); M. R. Perrone, Univ. del Salento (Italy); A. Pietruczuk, Institute of Geophysics (Poland); M. Pujadas, Ctr. de Investigaciones Energéticas, Medioambientales y Tecnológicas (Spain); J.-P. Putaud, European Commission Joint Research Ctr. (Italy); F. Ravetta, Univ. Pierre et Marie Curie (France); V. Rizi, Univ. degli Studi dell'Aquila (Italy); I. Serikov, Max-Planck-Institut für Meteorologie (Germany); M. Sicard, Univ. Politècnica de Catalunya (Spain); V. Simeonov, Ecole Polytechnique Fédérale de Lausanne (Switzerland); N. Spinelli, Consorzio Nazionale Interuniversitario per le Scienze Fisiche della Materia (Italy); K. Stebel, Norwegian Institute for Air Research (Norway); T. Trickl, Karlsruher Institut für Technologie (Germany); U. Wandinger, Leibniz Institut für Troposphärenforschung (Germany); X. Wang, Consorzio Nazionale Interuniversitario per le Scienze Fisiche della Materia (Italy); F. Wagner, Univ. de Évora (Portugal); M. Wiegner, Ludwig-Maximilians-Univ. München (Germany)

7832 OK Eyjafjallajökull volcano ash plume detection in the frame of the new constituting lidar network Leonet [7832-18]

S. Lolli, Leosphere France (France); S. Conil, Andra (France); A. Dabas, Meteo France (France); D. Donovan, KNMI (Netherlands); S.-E. Gryning, Risø National Lab. (Denmark); T. Mikkelsen, H. Ricketts, Univ. of Manchester (United Kingdom); L. Sauvage, Leosphere France (France); G. Vaughan, Univ. of Manchester (United Kingdom); J. Walter, Univ. Leipzig (Germany); F. Wienhold, ETH Zurich (Switzerland)

$7832 \mathrm{OL} \quad$ Lidar observations of the Eyjafjallajökull volcanic ash plume at Leipzig, Germany [7832-19] M. Tesche, A. Ansmann, A. Hiebsch, I. Mattis, J. Schmidt, P. Seifert, U. Wandinger, Leibniz Institut für Troposphärenforschung (Germany)

7832 OM Characterization of the Eyjafjallajökull ash-plume by means of lidar measurements over the Munich EARLINET-site [7832-20]

S. Groß, J. Gasteiger, V. Freudenthaler, F. Schnell, M. Wiegner, Ludwig-Maximilians-Univ. München (Germany)

7832 ON Mix of volcanic ash and Saharan dust over Romania during Eyjafjallajökull eruption [7832-21]

D. Nicolae, A. Nemuc, L. Belegante, National Institute of Research and Development for Optoelectronics (Romania) 
783200 EARLINET observations of the Eyjafjallajökull ash plume over Greece [7832-22]

D. Balis, E. Giannakaki, Aristotle Univ. of Thessaloniki (Greece); R. E. Mamouri, P. Kokkalis,

A. Papayannis, G. Tsaknakis, National Technical Univ. of Athens (Greece)

7832 OP Airborne measurements of the Eyjafjallajökull volcanic ash plume over northwestern Germany with a light aircraft and an optical particle counter: first results [7832-23] K. Weber, A. Vogel, C. Fischer, G. van Haren, T. Pohl, Univ. of Applied Sciences, Düsseldorf (Germany)

78320 Characterization of Iceland volcanic aerosols by UV-polarization lidar at Lyon, SW Europe [7832-24]

A. Miffre, G. David, B. Thomas, P. Rairoux, Lab. de Spectrométrie lonique et Moléculaire, CNRS, Univ. Claude Bernard Lyon 1 (France)

$7832 \mathrm{OR}$ Temporal and spatial structure of a volcanic ash cloud: ground-based remote sensing and numerical modeling [7832-25]

K. Schäfer, Karlsruher Institut für Technologie (Germany); W. Birmili, Leibniz Institut für Troposphärenforschung (Germany); J. Cyrys, Helmholtz Zentrum München GmbH (Germany); S. Emeis, R. Forkel, Karlsruher Institut für Technologie (Germany); S. Gilge, Deutscher Wetterdienst (Germany); C. Münkel, Vaisala GmbH (Germany); M. Pitz, Helmholtz Zentrum München GmbH (Germany); L. Ries, Umweltbundesamt (Germany); P. Suppan, Karlsruher Institut für Technologie (Germany)

\section{POSTER SESSION}

7832 OT Research on the nonlinearity correction method for the piezoelectric optical scanner in a lidar system [7832-34]

X. Wu, Huazhong Univ. of Science and Technology (China); S. Chen, Huazhong Univ. of Science and Technology (China) and Wuhan National Lab. for Optoelectronics (China); X. Xiong, B. Shi, W. Chen, Huazhong Univ. of Science and Technology (China)

7832 OU Scanning mobile lidar for aerosol tracking and biological aerosol identification [7832-30] T.-Y. He, F. Gao, S. Stanič, D. Veberič, K. Bergant, Univ. of Nova Gorica (Slovenia); A. Dolžan, Optotek (Slovenia); X.-Q. Song, Univ. of Nova Gorica (Slovenia) and Ocean Univ. of China (China)

7832 OV The study of atmospheric correction of satellite remotely sensed images intended for air pollution using sun-photometers (AERONET) and lidar system in Lemesos, Cyprus [7832-31]

D. G. Hadjimitsis, K. Themistocleous, A. Nisantzi, A. Matsas, Cyprus Univ. of Technology (Cyprus)

7832 OX Planetary boundary layer (PBL) monitoring by means of two laser radar systems: experimental results and comparison [7832-33] C. Bellecci, Univ. degli Studi di Roma Tor Vergata (Italy) and CRATI s.c.r.I., Univ. della Calabria (Italy); P. Gaudio, M. Gelfusa, A. Malizia, M. Richetta, C. Serafini, P. Ventura, Univ. degli Studi di Roma Tor Vergata (Italy)

Author Index 


\title{
Conference Committee
}

\author{
Symposium Chair
}

Steven P. Neeck, NASA Headquarters (United States)

Symposium Cochair

Karin Stein, Fraunhofer-Institut für Optronik, Systemtechnik und Bildauswertung (Germany)

\section{Conference Chairs}

Upendra N. Singh, NASA Langley Research Center (United States)

Gelsomina Pappalardo, Istituto di Metodologie per I'Analisi

Ambientale, CNR (Italy)

Program Committee

Arnoud Apituley, Rijksinstituut voor Volksgezondheid en Milieu (Netherlands)

Andreas Behrendt, Universität Hohenheim (Germany)

Gerhard Ehret, Deutsches Zentrum für Luft- und Raumfahrt e.V. (Germany)

Martin J. Endemann, European Space Research and Technology Center (Netherlands)

Pierre Henri Flamant, Laboratoire de Météorologie Dynamique (France)

Barry Gross, The City College of New York (United States)

Philippe L. Keckhut, Université de Versailles Saint-Quentin-en Yvelines (France)

Eduardo Landulfo, Instituto de Pesquisas Energéticas e Nucleares (Brazil)

Gennadii G. Matvienko, Institute of Atmospheric Optics (Russian Federation)

Doina Nicoleta Nicolae, National Institute of Research and Development for Optoelectronics (Romania)

Alexandros D. Papayannis, National Technical University of Athens (Greece)

Vincenzo Rizi, Università degli Studi dell'Aquila (Italy)

Laurent Sauvage, Leosphere France (France)

Valentin B. Simeonov, Ecole Polytechnique Fédérale de Lausanne (Switzerland)

Ulla Wandinger, Leibniz-Institut für Troposphärenforschung e.V. (Germany)

David M. Winker, NASA Langley Research Center (United States) 


\section{Session Chairs}

1 Lidar Techniques

Upendra N. Singh, NASA Langley Research Center (United States)

Fabio Madonna, Istituto di Metodologie per l'Analisi Ambientale, CNR (Italy)

2 Space-Based Lidar

Upendra N. Singh, NASA Langley Research Center (United States)

Fabio Madonna, Istituto di Metodologie per l'Analisi Ambientale, CNR (Italy)

3 Water Vapor, Aerosol, and Clouds

Eduardo Landulfo, Instituto de Pesquisas Energéticas e Nucleares (Brazil)

Doina Nicoleta Nicolae, National Institute of Research \& Development for Optoelectronics (Romania)

Joint Session with Conference 7827: Lidar Measurements during Recent 2010 Eyjafjallajökull Volcanic Eruption

Upendra N. Singh, NASA Langley Research Center (United States)

Adolfo Comeron, Universitat Politècnica de Catalunya (Spain)

Klaus Schäfer, Karlsruher Institut für Technologie (Germany) 